\title{
Primary Breast Tumors with Mesenchymal Morphology
}

\author{
Manvir S. Tevatia ${ }^{1}$ Prabhashankar Mishra ${ }^{1}$ Ajay K. Baranwal ${ }^{1}$ Prachi B. Nichat ${ }^{2, \odot ~ D i v y a ~ S h e l l y ~}{ }^{3}$ \\ Shivali Awasthi ${ }^{3}$ Prashant Sengupta ${ }^{1}$
}

${ }^{1}$ Department of Pathology, Command Hospital, Pune, Maharashtra, India

2Department of Pathology, Military Hospital, Secunderabad, Maharashtra, India

${ }^{3}$ Department of Pathology, Armed Forces Medical College, Pune, Maharashtra, India

\begin{abstract}
Address for correspondence Prachi Bhaurao Nichat, MBBS, MD, Department of Pathology, MH Secunderabad, Army Medical Corps, 1403, Surya Tower, near Tulshidham, Ghodbunder Road, Thane West, Maharashtra, 400607, India (e-mail: prachibnichat@gmail.com).
\end{abstract}

J Lab Physicians 2021;13:362-367.

\begin{abstract}
Keywords

- metaplastic

- mesenchymal

- sarcoma

- epithelial-mesenchymal transition

Overview Mesenchymal tumors of the breast are rare. Few epithelial tumors also have mesenchymal components. It is crucial to identify these as per histogenesis. This can be facilitated by markers of epithelial-mesenchymal transition (EMT).

Objectives The aim of this study was to categorize the breast lesions with mesenchymal morphology and to study EMT on immunohistochemistry (IHC).

Materials and Methods This is a retrospective study of 5-year duration from January 2015 to December 2019. Inclusion criteria: all breast lesions showing mesenchymal/nonepithelial morphology, complete or partial, on histology. Exclusion criteria: Mammary carcinomas without any mesenchymal/nonepithelial morphology, fibroadenomas, and lymphomas. Demographics, clinical, gross examination, histology, and IHC findings of selected cases were reviewed and recorded. Three additional markers $\mathrm{p} 53$, E-cadherin, and $\beta$-catenin were performed.

Statistical Analysis Used Frequency calculation for each variable (IHC).

Results Thirteen (2.5\%) out of total 510 breast specimens showed mesenchymal histology. Of these, five (38.5\%) were metaplastic breast carcinomas (MBC), four (31\%) were phyllodes tumor (PT), and one (7.7\%) case each of malignant peripheral nerve sheath tumor, primary stromal sarcoma of breast, pseudoangiomatous stromal hyperplasia, and myofibroblastoma. Loss of E-cadherin was seen in 4/5 (80\%) MBCs and was retained in ductal component of PTs. p53 was not expressed in any of the tumors except $3 / 5(60 \%)$ MBCs. $\beta$-Catenin was aberrant in all MBCs.

Conclusions Primary breast tumors with mesenchymal morphology present a spectrum ranging from benign mesenchymal, fibroepithelial neoplasms to malignant tumors of mesenchymal and epithelial origin. Loss of E-cadherin, expression of p53, and aberrant expression of $\beta$-catenin are suggestive of EMT and molecular heterogeneity of MBCs.
\end{abstract}

published online July 9,2021
DOI https://doi.org/

$10.1055 / \mathrm{s}-0041-1732492$ ISSN 0974-2727
C 2021. The Indian Association of Laboratory Physicians.

This is an open access article published by Thieme under the terms of the Creative Commons Attribution-NonDerivative-NonCommercial-License, permitting copying and reproduction so long as the original work is given appropriate credit. Contents may not be used for commercial purposes, or adapted, remixed, transformed or built upon. (https://creativecommons.org/licenses/by-nc-nd/4.0/).

Thieme Medical and Scientific Publishers Pvt. Ltd. A-12, 2nd Floor, Sector 2, Noida-201301 UP, India 


\section{Introduction}

Mesenchymal neoplasms comprise 0.5 to $1 \%$ of all breast tumors. ${ }^{1,2}$ Malignant mesenchymal breast tumors are aggressive, have propensity to recur, and bear a poor prognosis. Although the World Health Organization classification of breast neoplasms ${ }^{3}$ iterates different histological subtypes depending on cell morphology, it is challenging to differentiate between epithelial and mesenchymal tumors of breast owing to the rarity of the latter and presence of entities like metaplastic carcinomas that exhibit heterogenous morphology.

The clinical and pathological attributes of these rare neoplasms have been sparsely studied, especially in resource-limited countries like ours. With the rapid advent of molecular and genetic diagnostic tools, our knowledge of the pathogenetic underpinnings of breast neoplasms is expanding every day. TP53 and CTNNB1 mutations among others (like EGFR, PIK3CA, PTEN) have been hypothesized to play a significant role in epithelial-mesenchymal transition (EMT) in metaplastic breast carcinomas (MBCs) ${ }^{4-6}$

This study is an attempt to categorize the breast lesions that share mesenchymal morphology emphasizing on the diagnostic characteristics on histology and to study EMT with immunohistochemistry (IHC) markers such as p53, $\beta$-catenin, and E-cadherin.

\section{Materials and Methods}

\section{Samples}

This is a retrospective study of 5-year duration from January 2015 to December 2019. We included all reported mastectomies, lumpectomies, excision biopsies as well as core needle biopsies of breast lesions that exhibited mesenchymal/nonepithelial morphology, completely or partially. Mammary carcinomas without any mesenchymal/nonepithelial morphology, fibroadenomas, and lymphomas were not included.
The age, clinical details, radiology, gross examination findings, histomorphology, and IHC were reviewed, analyzed, and recorded.

\section{Immunohistochemistry}

Three additional immunohistochemical markers namely, p53 (clone BP-53-12, PathnSitu, Cat \#CM101), E-cadherin (clone EP6, PathnSitu, Cat\#CR039) and $\beta$-catenin (clone EP35, PathnSitu, Cat\#CR005) were performed on representative formalin fixed paraffin blocks. Sections of $3 \mu \mathrm{m}$ were applied on poly-L-lysine coated slides and incubated for 24 hours. Deparaffinization was facilitated with 3 changes of xylene and hydration in graded series of alcohol. Antigen retrieval was achieved in Decloaker at medium pressure for 7 minutes in citrate buffer ( $\mathrm{pH}-6.0)$. After washing in distilled water (DW), tissue was incubated with $\mathrm{H}_{2} \mathrm{O}_{2}$ for 10 minutes to block endogenous peroxidase activity and rinsed with tris-buffered saline (TBS). Primary antibody was applied and incubated in moist chamber for 1 hour at room temperature. Slides were washed with TBS, treated with PolyExcel Target binder (PathnSitu, Hyderabad, Telangana, India) for 10 minutes and washed again to be incubated with PolyExcel Horse radish peroxidase (PathnSitu, Hyderabad, Telangana, India) for 15 minutes. Subsequently, tissue was treated with 3,3'-diaminobenzidine substrate chromogen for 5 minutes and washed in DW. These slides were then counterstained with hematoxylin, dehydrated, cleared, and mounted. Known controls were used for $\mathrm{p} 53$, while $\beta$-catenin and E-cadherin were assessed with internal controls (endothelial cells and duct epithelium, respectively). Frequency of positive cases was calculated for each of the above IHC markers.

\section{Interpretation of IHC}

p53 was considered positive if strong nuclear positivity was noted in more than $10 \%$ tumor cells after examining at least five high power fields or 1,000 tumor cells. ${ }^{7.8}$ Membranous staining of cells was considered positive for E-cadherin. The

Table 1 Details of metaplastic breast carcinomas

\begin{tabular}{|c|c|c|c|c|c|}
\hline Case & Specimen received & Radiology findings & Gross examination & $\begin{array}{l}\text { Metaplastic } \\
\text { component }\end{array}$ & $\mathrm{IHC}$ \\
\hline 1 & Lumpectomy & $\begin{array}{l}\text { Well circumscribed } \\
\text { solid cystic lesion with } \\
\text { foci of calcification-BI- } \\
\text { RADS IV }\end{array}$ & $\begin{array}{l}3 \times 2 \times 1.5 \mathrm{~cm} \text { solid } \\
\text { cystic tumor with hard } \\
\text { consistency }\end{array}$ & $\begin{array}{l}\text { Osteosarcoma and } \\
\text { angiosarcoma }\end{array}$ & $\begin{array}{l}\text { ER(-), PR(-), Her2 } \\
\text { neu(-), p63(-), CK }(+), \\
\text { FLI(-), CD10(-), S100(-), } \\
\text { CD34(-), } \\
\text { Mib 1-25\% }\end{array}$ \\
\hline 2 & Lumpectomy & NA & $\begin{array}{l}14 \times 8 \times 7 \mathrm{~cm} \text { fungating } \\
\text { growth with solid areas } \\
\text { and cystic change }\end{array}$ & Spindle cell & $\begin{array}{l}\text { ER(-), PR(-), Her2 neu(-), } \\
\text { Mib1- 18-20\% }\end{array}$ \\
\hline 3 & Trucut biopsy & NA & NA & Spindle cell & $\begin{array}{l}\text { ER(-), PR(-), Her } 2 \text { neu(-), } \\
\text { CK(+), Vimentin(+) }\end{array}$ \\
\hline 4 & $\begin{array}{l}\text { Paraffin blocks for } \\
\text { review }\end{array}$ & NA & NA & $\begin{array}{l}\text { Spindle cell, } \\
\text { squamous }\end{array}$ & $\begin{array}{l}\text { ER(-), PR(-), Her } 2 \text { neu(-), } \\
\text { HMWCK(+), p63(+) }\end{array}$ \\
\hline 5 & $\begin{array}{l}\text { Paraffin blocks for } \\
\text { review }\end{array}$ & NA & NA & Spindle cell & $\begin{array}{l}\mathrm{ER}(-), \operatorname{PR}(-), \text { Her2neu(-), } \\
\mathrm{CK}(+), \operatorname{Vimentin}(+), \\
\operatorname{EMA}(+), \operatorname{SMA}(), \mathrm{K}_{\mathrm{i}} 67-70 \%\end{array}$ \\
\hline
\end{tabular}

Abbreviations: BIRADS IV, Breast Imaging Reporting and Data System IV; IHC, immunohistochemistry; NA, not available. 
expression of $\beta$-catenin was noted as normal or aberrant. Strong membranous staining was considered normal, while aberrant expression was classified as:

- Complete loss of membranous (LOM) without cytoplasmic/nuclear staining.

- Partial LOM without cytoplasmic/nuclear staining.

\section{Results}

A total of 510 breast specimens were received for histopathological examination from January 2015 to December 2019. Of these, thirteen (2.5\%) cases were found to have the histology showing mesenchymal morphology partially or completely. As proven by gross, histomorphology, and IHC examination, these included five cases of MBCs, four cases of phyllodes tumors (PTs), one case each of malignant peripheral nerve sheath tumor (MPNST), primary spindle cell sarcoma of breast, myofibroblastoma, and pseudoangiomatous stromal hyperplasia. Age of the 12 female patients ranged from 23 to 52 years while that of one included male patient was 85 years. The mean age of the participants was 44 years with a standard deviation (SD) of 14.89 .
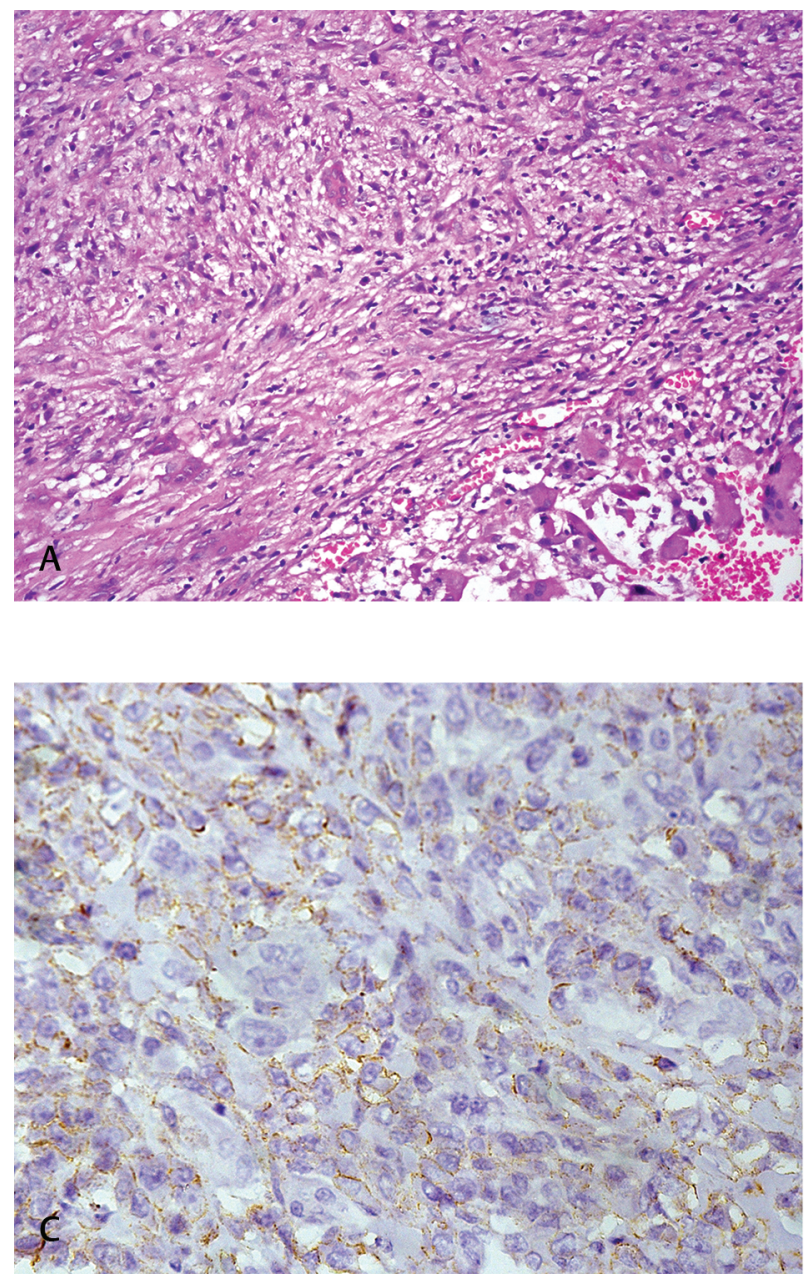

\section{Metaplastic Carcinomas}

Of the five MBC cases reported, two were received as lumpectomies, two as paraffin blocks for review consultation, and one as trucut biopsy. The details are described in - Table 1. The mean age was 43.20 years $(S D=7.02)$. All five cases showed presence of tumor necrosis, while osteoclast like multinucleated giant cells were seen in four out of five cases. Though the mesenchymal component ( - Fig. 1A) was initially not graded in the previous reports, on review by the authors, in all the cases it was found to be of high histological grade.

\section{Phyllodes Tumors}

Four cases of PT of breast were reported, of which one was benign, one was borderline, and two were malignant. Age of the patients ranged from 34 to 48 years, mean age being 40 years $(S D=5.17)$. One case was received for review and the rest as wide local excision specimens in which the tumors ranged grossly from 4.5 to $12 \mathrm{~cm}$ in largest dimensions. One of the malignant tumors was accompanied with an axillary lump with similar histology measuring $2.5 \mathrm{~cm}$. p53 was not expressed in any of the PTs. E-cadherin and $\beta$-catenin, though normally expressed in the ductal component, showed no expression in the stromal component.
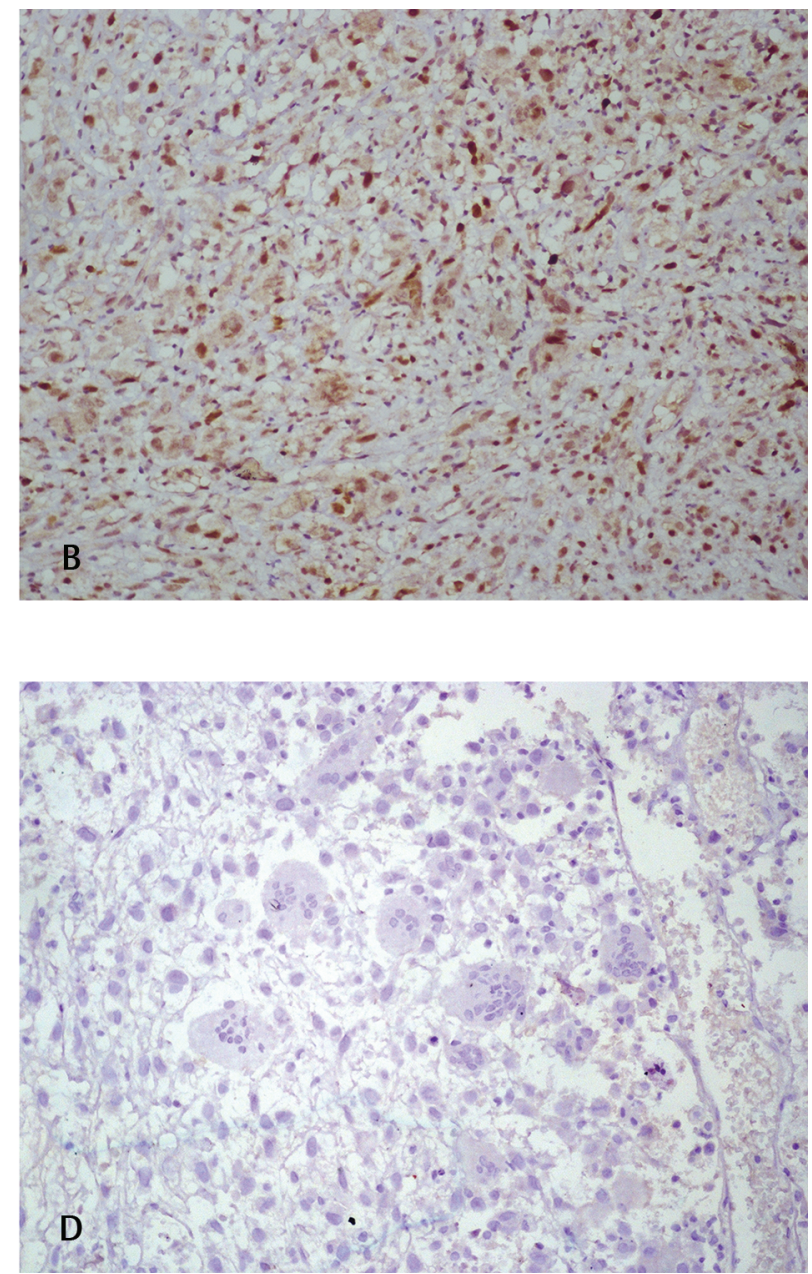

Fig. 1 (A) Metaplastic breast carcinoma (MBC) showing spindle cell morphology (Hematoxylin and Eosin, 200×). (B) Strong nuclear p53 positivity in MBC (200x). (C) Partial loss of membranous staining of $\beta$-catenin in MBC (200×). (D) Complete loss of E-cadherin in MBC (200×). 


\section{Primary Sarcomas of the Breast}

Two cases of sarcoma were reported. Trucut biopsy was reported initially, one was reported as fibrosarcoma breast and the other as metaplastic carcinoma. This was followed by modified Radical mastectomy with axillary dissection in both cases.

The former a 30-year-old female presented with recurrent left breast lump. There was history of previous lumpectomy diagnosed as malignant phyllodes elsewhere. Positron emission tomography scan showed fluorodeoxyglucose avid enhancing solid mass lesion in retroareolar region. Mastectomy specimen showed two tumor masses measuring 6 and $7 \mathrm{~cm}$ with white firm to hard cut surface both ulcerating the skin. Histology showed spindle cells arranged in short, intersecting fascicles with alternating hypocellular areas. On IHC, tumor cells were CK \& EMA(-), Vimentin(+), CD10(+), S100(+), CD34(-), Desmin(-), SMA(focal), c-Kit(-), p63(-), CD99(-), CD31(-), ER(-), PR(-), and Her 2 neu(-). All 14 lymph nodes were free from tumor. The final diagnosis was revised as MPNST, high grade, in view of histomorphology and IHC findings.

The latter, a 23-year-old female, presented with a left breast lump. Grossly, a tumor measuring $12 \mathrm{~cm}$ with firm gray white cut surface was noted. Radiology findings were not available in this case. Histology showed spindle cells arranged in loose intersecting fascicles with abundant eosinophilic cytoplasm and pleomorphic nuclei with nucleoli. Few cells had epithelioid appearance. On IHC, tumor cells were CK \& EMA(-), Vimentin(+), CD10(+), SMA(+), S100(-), Desmin(-), Myogenin(-), CD 31(-), CD34(-), HMB 45(-), $\operatorname{HMWCK}(-), \operatorname{LMWCK}(-), \mathrm{p} 63(-)$, and c-Kit(-). All 23 lymph nodes were free from tumor. The diagnosis offered was primary spindle cell sarcoma of the breast.

Both the cases showed extensive areas of necrosis and presence of tumor giant cells. p53, E-cadherin, and $\beta$-catenin were not expressed in either of these cases.

\section{Benign Tumors}

A lumpectomy specimen of a 62-year-old female with radiology findings of ill-defined high-density lesion and Breast Imaging Reporting and Data System (BIRADS) score V was received. Gross examination showed a tumor measuring $4 \mathrm{~cm}$ with gray white firm to hard cut surface. Histology showed proliferation of fibroblastic stromal elements admixed with dilated breast ducts, stromal hyperplasia with dense keloid like quality, and anastomosing pattern of slit like clefts lined by single layer of spindle cells simulating blood vessels. The final diagnosis was pseudoangiomatous stromal hyperplasia. Due to nonavailability of paraffin blocks, additional IHC was not performed.

A trucut biopsy from left breast in an 85-year-old male was received. Ultrasonography showed a $4 \mathrm{~cm}$ nodule in left breast, but BIRADS score was not mentioned. The 04 linear tissue cores with largest measuring $1.2 \mathrm{~cm}$ showed a spindle cell tumor disposed in relatively hypercellular fascicles and whorls along with inflammatory cells like mast cells, eosinophils, plasma cells, and lymphocytes on histology. Tumor cells were Vimentin(+), SMA(+), CD34(+), Desmin(+), S100(-),
HMB45(-), and Mib 2\%. It was diagnosed as myofibroblastoma. None of the additional IHC markers were expressed.

\section{Discussion}

Breast parenchyma is composed of two major lobular components-ducts and the periductal stroma. Besides these, interlobular hormone responsive stroma, the supportive structure for the terminal duct lobular units is the third component. This physiology gives rise to biphasic pathologies and the problem arises when the stromal component is predominantly involved. This coupled with decreasing size of biopsies poses a challenge, one which can only be mitigated by collating the clinical, histological and IHC findings to determine the histogenesis of the mesenchymal component. Whereas epithelial tumors of the breast have multipronged treatment strategies, the management of phyllodes and sarcomas begins with surgical excision and negative margins. Moreover, sarcomas have a poorer prognosis than PT with an overall 5-year survival rate ranging from 50 to $90 \%$ depending on tumor size. ${ }^{9,10}$

Over 5-year duration, we found 2.5\% tumors with mesenchymal morphology, a small but sizeable number. Primary sarcomas of breast are rare accounting for $<1 \%$ of all breast neoplasms. MPNSTs are extremely rare soft tissue sarcomas ( $10 \%$ of soft tissue tumors and incidence of $0.001 \%$ in general population), especially with primary in breast. ${ }^{11}$ They are often associated with Von Recklinghausen neurofibromatosis. The mean age at diagnosis is 35 years. ${ }^{12,13}$ In our case, the lady was young, 30 years of age, presented with a recurrent rapidly growing large tumor, previously diagnosed as malignant phyllodes. Another rare entity in our study, a case of primary stromal sarcoma of breast, was diagnosed in a 23-year-old female. Stromal sarcomas are malignant mesenchymal tumors arising from hormone-sensitive specialized stroma of mammary gland and tumor cells characteristically express CD10. ${ }^{14}$

It is essential to perform extensive sampling to ascertain or rule out presence of leaf like architecture, epithelial component in the form of clefts/hyperplasia (PTs), or islands of metaplasia/epithelioid cells (MBCs). IHC markers for neuron-specific enolase, Vimentin, and S-100 are instrumental in confirming the diagnosis in MPNST, while Ki 67 proliferation index helps to rule out benign neural tumors. Vimentin and CD10 positivity after excluding other cells of origin are diagnostic for stromal sarcomas.

PTs are derived from periductal stroma and account for approximately 0.3 to $1 \%$ of all breast tumors. ${ }^{15}$ The diagnosis is based on the assessment of stromal cellularity, mitotic activity, and the microscopic character of the tumor border. Although some studies ${ }^{16}$ have shown that p53 expression significantly separates benign from malignant PTs, none of the PTs in our study showed significant p53 expression. E-cadherin and $\beta$-catenin expression was comparable to control in all PTs.

Metaplastic carcinomas are complex neoplasms showing variability in phenotype. MBCs are also notorious for high rate of metastasis and are triple negative tumors with poor 
prognosis. Studies show that the different histologies, especially spindle cell transformation of the tumor, are the result of genetic alteration rather than shift in histogenesis. ${ }^{17}$ EGFR gene amplification, TP53 mutations, and CTNNB1 mutations (through Wnt canonical pathway) have been implicated in EMT that, in turn, decreases cell to cell adhesion and increases propensity to metastasize. Loss of E-cadherin seen in $80 \%$ MBCs in our study is a marker of EMT. ${ }^{18}$ We used p53 and $\beta$-catenin as surrogate markers to study the pathways (induced by TP53 and CTNNB1 mutations) involved in EMT. p53 was expressed in $60 \%$ cases, consistent with similar studies on TP53 mutational analysis. ${ }^{3}$

In colorectal or pancreaticobiliary carcinomas, nuclear localization of $\beta$-catenin is suggestive of $\beta$-catenin stabilization by activation of Wnt signaling pathway. In our study, none of the cases showed nuclear localization. $\beta$-Catenin expression was aberrant in all cases but with LOM staining without cytoplasmic/nuclear staining. This leads us to believe that activation of Wnt signaling pathway in breast carcinomas is induced by somatic mutations other than APC, CTNNB1, FAM123B, etc. seen in colorectal cancers., ${ }^{4,18,19}$ Studies suggest that this type of aberration may implicate other molecular pathways and is associated with tumor invasiveness, lymph node metastasis, and poor prognosis. ${ }^{20}$

It is worthy of mentioning that $80 \%$ MBCs showed presence of osteoclast like multinucleated giant cells, although their significance is unknown. Although rarity of these cases remains an obstacle, further preferably multicentric studies are required to better elucidate the molecular and genetic characteristics of these tumors.

Myofibroblastomas, though rare, are the commonest benign mesenchymal neoplasm of breast after haemangiomas. ${ }^{3}$ They are known to affect elderly males and postmenopausal women. It is challenging to diagnose on core biopsy since they can mimic both ductal and mesenchymal malignancies. But, lack of necrosis, nuclear atypia, and mitoses compounded with characteristic nuclear and stromal features should nudge one into excluding myofibroblastic origin.

\section{Conclusion}

Primary breast tumors with mesenchymal morphology are rare and present a wide spectrum of neoplasms ranging from benign mesenchymal, biphasic fibroepithelial neoplasms to malignant tumors of mesenchymal as well as epithelial origin. Malignant mesenchymal tumors are much rarer and require a high index of suspicion for their diagnosis after diligently excluding commoner entities. Loss of E-cadherin in triple negative breast carcinomas should prompt a search for mesenchymal component (MBCs) indicative of EMT. Expression of p53 and aberrant (LOM) expression of $\beta$-catenin are exclusive to MBCs among other mesenchymal tumors and are indicative of the molecular heterogeneity in MBCs through involvement of various pathways, waiting to be explored through further molecular analysis.

\section{Statement of Conforming to the Declaration of Helsinki}

This is a retrospective study and does not involve any intervention or human participants. For this type of study, formal consent is not required as all personal identification data are anonymized. We, the authors, have performed the research in accordance with the ethical standards as laid down in the 1964 Declaration of Helsinki.

\section{Authors' Contribution}

Manvir S Tevatia was involved in concept, design, definition of intellectual content, data analysis, statistical analysis, manuscript editing, and manuscript review. Prabhashankar Mishra was involved in concept, design, definition of intellectual content, data analysis, statistical analysis, manuscript preparation, and manuscript review. Ajay K Baranwal was involved in concept, design, definition of intellectual content, data analysis, manuscript preparation, and manuscript review. Prachi B Nichat was involved in concept, design, definition of intellectual content, literature research, data acquisition, statistical analysis, manuscript preparation, and manuscript review. Divya Shelly was involved in concept, design, definition of intellectual content, data analysis, statistical analysis, manuscript editing and review. Shivali Awasthi was involved in concept, design, definition of intellectual content, literature research, data acquisition, and manuscript review. Prashant Sengupta1 was involved in concept, design, definition of intellectual content, data analysis, statistical analysis, and manuscript editing and review. Prachi B Nichat is guarantor for this manuscript.

\section{Conflicts of Interest}

Nil.

\section{References}

1 O'Donnell ME, McCavert M, Carson J, Mullan FJ, Whiteside MW, Garstin WI. Non-epithelial malignancies and metastatic tumours of the breast. Ulster Med J 2009;78(2):105-112

2 Young JL Jr, Ward KC, Wingo PA, Howe HL. The incidence of malignant non-carcinomas of the female breast. Cancer Causes Control 2004;15(3):313-319

3 Goldblum JR, Lazar AJ, Schnitt SJ, Tan PH, Mesenchymal tumours of the Breast. In: WHO Classification of Tumours Editorial Board. Breast Tumours. 5th edition. Lyon, France: International Agency for Research on Cancer; 2019 187-230

4 Schwartz TL, Mogal H, Papageorgiou C, Veerapong J, Hsueh EC. Metaplastic breast cancer: histologic characteristics, prognostic factors and systemic treatment strategies. Exp Hematol Oncol 2013;2(1):31

5 Lacroix-Triki M, Geyer FC, Lambros MB, et al. $\beta$-catenin/Wnt signalling pathway in fibromatosis, metaplastic carcinomas and phyllodes tumours of the breast. Mod Pathol 2010;23(11):1438-1448

$6 \mathrm{Ng}$ CKY, Piscuoglio S, Geyer FC, et al. The landscape of somatic genetic alterations in metaplastic breast carcinomas. Clin Cancer Res 2017;23(14):3859-3870

7 Lee SK, Bae SY, Lee JH, et al. Distinguishing low-risk luminal a breast cancer subtypes with ki-67 and p53 is more predictive of long-term survival. PLoS One 2015;10(8):e0124658 
8 van der Kooy K, Rookus MA, Peterse HL, van Leeuwen FE. p53 protein overexpression in relation to risk factors for breast cancer. Am J Epidemiol 1996;144(10):924-933

9 Adem C, Reynolds C, Ingle JN, Nascimento AG. Primary breast sarcoma: clinicopathologic series from the Mayo Clinic and review of the literature. Br J Cancer 2004;91(2):237-241

10 Pollard SG, Marks PV, Temple LN, Thompson HH. Breast sarcoma. A clinicopathologic review of 25 cases. Cancer 1990;66(5):941-944

11 Weiss SW, Goldblum JR, Malignant tumors of the peripheral nerves. In: Strauss M, Grey L, eds. Enzinger and Weiss's Soft Tissue Tumors. 4th edition. St. Louis: Mosby Inc; 2001 1209-1264

12 Ducatman BS, Scheithauer BW, Piepgras DG, Reiman HM, IlstrupDM. Malignantperipheralnervesheath tumors. Aclinicopathologic study of 120 cases. Cancer 1986;57(10):2006-2021

13 Dhingra KK, Mandal S, Roy S, Khurana N. Malignant peripheral nerve sheath tumor of the breast: case report. World J Surg Oncol 2007;5:142

14 Kumar S, Sharma J, Ralli M, Singh G, Kalyan S, Sen R. Primary stromal sarcoma of breast: a rare entity. Iran J Pathol 2016;11(5):469-473
15 Tse G, Koo JS, Thike AA, Phyllodes tumours. In: WHO Classification of Tumours Editorial Board. Breast Tumours. 5th edition. Lyon, France: International Agency for Research on Cancer; 2019 172-176

16 Gatalica Z, Finkelstein S, Lucio E, et al. p53 protein expression and gene mutation in phyllodes tumors of the breast. Pathol Res Pract 2001;197(3):183-187

17 Hoda SA, Brogi E, Koerner FC, Rosen PP, Rosen's Breast Pathology. 4th edition. New York: Lippincott, Williams \& Wilkins; 2014 547-599

18 Zhang Y, Toy KA, Kleer CG. Metaplastic breast carcinomas are enriched in markers of tumor-initiating cells and epithelial to mesenchymal transition. Mod Pathol 2012;25(2):178-184

19 Geyer FC, Weigelt B, Natrajan R, et al. Molecular analysis reveals a genetic basis for the phenotypic diversity of metaplastic breast carcinomas. J Pathol 2010;220(5):562-573

20 Lee WA. Prognostic significance of abnormal $\beta$-catenin expression in breast carcinoma.J Korean Med Sci 2005;39(2):114-119 